\title{
DIMENSIONAMENTO DE PESSOAL DE ENFERMAGEM NA UTI-ADULTO DE HOSPITAL UNIVERSITÁRIO PÚBLICO
}

\author{
Fabieli Borges ${ }^{1}$, Cristina Daiana Bohrer ${ }^{2}$, Thais Vanessa Bugs ${ }^{2}$, Anair Lazzari Nicola ${ }^{3}$, Nelsi Salete Tonini ${ }^{4}$, \\ João Lucas Campos de Oliveira ${ }^{5}$
}

\begin{abstract}
RESUMO: O objetivo foi dimensionar o quadro de pessoal de enfermagem de uma Unidade de Terapia Intensiva para adultos do Paraná e compará-lo com o quadro real existente. Pesquisa transversal, com uso de fonte documental. Os dados foram coletados sobre 122 dias ininterruptos prévios de junho a setembro de 2016 referentes às características sociodemográficas, clínicas e pontuação diária do Nursing Activities Score da amostra $(n=128)$ de pacientes; e aos trabalhadores do setor. Aos dados tabulados, procedeu-se análise estatística descritiva, aplicação de equação própria do dimensionamento de pessoal em terapia intensiva e ajuste do quantitativo dimensionado à categoria profissional segundo a nova Resolução do Conselho Federal de Enfermagem, $\mathrm{n}^{\circ}$ 543/2017. A média do Nursing Activities Score da unidade foi 1514,89. Na comparação do quadro dimensionado $(n=87)$ com o real $(n=60)$, houve um déficit de 38 enfermeiros e superávit de 11 técnicos de enfermagem. O quadro de pessoal real não corresponde à necessidade de enfermeiros.

DESCRITORES: Downsizing organizacional; Unidades de terapia intensiva; Equipe de enfermagem; Administração de recursos humanos; Carga de trabalho.

\section{NURSING STAFF DIMENSIONING AT THE ADULT ICU OF A PUBLIC TEACHING HOSPITAL}

ABSTRACT: The objective was to dimension the nursing staff at an Intensive Care Unit for adults in the state of Paraná and compare it with the existing staff. Cross-sectional research using a documentary source. The data were collected during 122 uninterrupted days between June and September 2016 - concerning the sociodemographic and clinical characteristics and daily Nursing Activities Score of the patient sample $(n=128)$; and concerning the professionals working at the sector. The collected data were subject to descriptive statistical analysis, application of an intensive care staff dimensioning equation and adjustment of the dimensioned staff to the professional category according to the new Resolution by the Brazilian Federal Nursing Council, No.543/2017. The average Nursing Activities Score at the unit was 1514.89. The comparison between the dimensioned $(n=87)$ and the actual staff $(n=60)$ revealed a shortage of 38 nurses and a surplus of 11 nursing technicians. The actual staff does not correspond to the need for nurses.

DESCRIPTORS: Personnel downsizing; Intensive care units; Nursing, team; Personnel management; Workload.
\end{abstract}

\section{DIMENSIONAMIENTO DE PERSONAL DE ENFERMERÍA EN LA UCI-ADULTO DE HOSPITAL UNIVERSITARIO PÚBLICO}

RESUMEN: El objetivo fue dimensionar el cuadro de personal de enfermería de una Unidad de Terapia Intensiva para adultos de Paraná y compararlo con el grupo real existente. Investigación trasversal con uso de fuente documental. Los datos fueron recolectados sobre 122 días ininterrumpidos de junio a septiembre del 2016 - referentes a las características sociodemográficas, clínicas y puntuación diaria del Nursing Activities Score de la muestra $(n=128)$ de pacientes; y a los trabajadores del sector. A los datos tabulados fue aplicado análisis estadístico descriptivo, aplicación de ecuación propia del dimensionamiento de personal en terapia intensiva y ajuste de la cuantidad dimensionada a la categoría profesional según la nueva Resolución del Consejo Federal de Enfermería, nº 543/2017. El promedio de la Nursing Activities Score de la unidad fue 1514.89. En la comparación del cuadro dimensionado $(n=87)$ y real $(n=60)$, fue encontrado un déficit de 38 enfermeros y superávit de 11 técnicos de enfermería. El cuadro de personal real no corresponde a la necesidad de enfermeros.

DESCRIPTORES: Reducción de personal; Unidades de cuidados intensivos; Grupo de enfermería; Administración de personal; Carga de trabajo.

${ }^{1}$ Enfermeira. Mestranda em Enfermagem. Universidade Federal do Paraná. Curitiba, PR, Brasil.

${ }^{2}$ Enfermeira. Especialista em Gerenciamento de Enfermagem em Clínica Médica e Cirúrgica. Enfermeira Residente em Vigilância em Saúde e Controle de Infecções na Universidade Estadual do Oeste do Paraná. Cascavel, PR, Brasil.

${ }^{3}$ Enfermeira. Doutora em Enfermagem Fundamental. Docente da Universidade Estadual do Oeste do Paraná. Cascavel, PR, Brasil.

${ }^{4}$ Enfermeira. Doutora em Enfermagem Psiquiátrica. Docente da Universidade Estadual do Oeste do Paraná. Cascavel, PR, Brasil.

${ }^{5}$ Enfermeiro. Doutorando em Enfermagem. Universidade Estadual de Maringá. Maringá, PR, Brasil.

Autor Correspondente:

Recebido: $23 / 01 / 2017$

Fabieli Borges

Universidade Federal do Paraná

Finalizado: $25 / 05 / 2017$

R. Odontologia, 834 - 85819-220 - Cascavel, PR, Brasil.

E-mail: fabieliborges6@gmail.com 


\section{- INTRODUÇÃO}

A Unidade de Terapia Intensiva (UTI) desempenha papel fundamental para recuperação de pessoas com estado de saúde crítico. Sabidamente, é um setor de alta complexidade, característica verificada pela peculiaridade dos pacientes atendidos, que não raras vezes precisam de diversas intervenções diagnóstico-terapêuticas invasivas e complexas. Além do aparato tecnológico vinculado ao cuidado, o atendimento na UTI deve dispor de recursos humanos especializados capazes de aplicar os conhecimentos de forma segura, com vistas à melhor recuperação da clientela gravemente enferma ${ }^{(1)}$.

Em que pese o impacto do capital humano na qualidade do cuidado intensivo, cumpre destacar a importância da equipe de enfermagem, que representa o maior contingente de trabalhadores lotados neste e em outros setores hospitalares, bem como a única categoria profissional que permanece junto ao doente internado de forma ininterrupta ${ }^{(2)}$.

À liderança da equipe de enfermagem, a função do enfermeiro no cenário de uma UTI deve ser bem definida, pois além da assistência direta, ele deve responsabilizar-se sobre o aprimoramento dos profissionais; manejo de tecnologias; humanização; práticas interdisciplinares; e, não menos importante, o gerenciamento da equipe sob sua supervisão ${ }^{(3)}$.

Ainda que complexa, a gerência de enfermagem, inclusive na UTI, pode ser facilitada com uso de meios e instrumentos para tal fim, advindos essencialmente de conhecimentos administrativos que favorecem o trabalho gerencial do enfermeiro ${ }^{(3-4)}$. Neste aspecto, um dos instrumentos que deve ser utilizado pelo enfermeiro no bojo do gerenciamento de recursos humanos é o dimensionamento, que prevê o quantitativo e qualitativo de pessoal necessário para suprir as demandas de cuidado requeridas por uma dada clientela ${ }^{(5)}$.

No Brasil, por mais de dez anos o dimensionamento foi oficialmente orientado pelo Conselho Federal de Enfermagem (COFEN) por meio de resolução que, muito recentemente (maio de 2017), teve merecida e aclamada atualização ${ }^{(5)}$. Acredita-se que, por si só, este fato justifica a necessidade de novos estudos acerca do dimensionamento da enfermagem brasileira, uma vez que a atualização contempla mudanças na proporção de profissionais por pacientes e nas horas de enfermagem requeridas às categorias (graus de dependência) de cuidado ${ }^{(5)}$.

O dimensionamento é um método fundamentado em expressões matemáticas que se utiliza de variáveis inerentes à clientela, ao serviço de enfermagem e à organização, com amplo destaque à carga de trabalho da equipe de enfermagem em sua operacionalização(5-6). Em UTI, apesar de existir a necessidade de sua contínua revisão, o Nursing Activities Score (NAS) é amplamente utilizado e recomendado à mensuração da carga de trabalho da enfermagem intensivista, além de mediar o próprio dimensionamento de pessoal ${ }^{(7)}$.

No contexto de UTI, o aumento da carga de trabalho da equipe de enfermagem - comumente associado ao déficit no quadro de pessoal - têm apresentado resultados alarmantes ao cuidado dos pacientes, tais como: aumento da permanência na internação( ${ }^{(8)}$; elevação dos índices de infecções relacionadas à assistência, lesões por pressão, quedas e erros na administração de medicamentos ${ }^{(9)}$; e até mesmo a maior propensão à morte ${ }^{(10)}$.

Considerando a relevância da equipe de enfermagem na qualidade e segurança do atendimento em UTI, bem como da atualização recente da Resolução oficial que rege o dimensionamento de pessoal no Brasil, considera-se que investigar o tema em pauta é social e cientificamente relevante, uma vez que os resultados de pesquisas podem ser um subsídio formal e atualizado para possível readequação de pessoal.

Isso possivelmente poderá contribuir diretamente à qualidade do cuidado e segurança do paciente. Não menos importante, ressalta-se que os estudos recentes relacionam a carga de trabalho de enfermagem em UTI, e não o próprio dimensionamento como objeto de pesquisa ${ }^{(6-11)}$, o que reforça a justificativa para novas pesquisas.

Ante ao exposto, as inquietações para este estudo culminaram ao seguinte questionamento: $\mathrm{O}$ quadro dimensionado de pessoal de enfermagem em uma UTI-adulto corresponde ao real apresentado? 
A fim de responder a indagação, objetivou-se dimensionar o quadro de pessoal de enfermagem de uma Unidade de Terapia Intensiva para adultos do Paraná e compará-lo com o quadro real existente.

\section{MÉTODOS}

Pesquisa transversal, com uso de fonte documental e abordagem quantitativa. Foi realizada na UTIadulto de um hospital universitário público localizado no estado do Paraná, Brasil. O hospital conta com 210 leitos exclusivamente vinculados ao Sistema Único de Saúde (SUS), bem como atende uma população que abrange em torno dois milhões de habitantes, sendo referência à assistência ao trauma, gestação de alto risco e tratamento do Vírus da Imunodeficiência Humana (HIV).

A UTI pesquisada apresenta um total de 14 leitos, sendo que cinco são destinados para realização de hemodiálise, conforme a necessidade clínica dos pacientes atendidos. O trabalho da enfermagem na unidade é dividido entre cinco equipes para os turnos matutino, vespertino e três períodos noturnos.

A pesquisa se deu sobre a totalidade de pacientes internados e profissionais de enfermagem em labor em um período de 122 dias de observação prévia, correspondente aos meses de junho a setembro de 2016. A coleta de dados ocorreu em novembro de 2016. Para isso, utilizou-se a planilha de gerenciamento de enfermagem, documento formal da instituição. Ainda, foram compilados os dados referentes à caracterização sociodemográfica e clínica dos pacientes internados no recorte temporal, por meio de prontuário eletrônico que se vale de software terceirizado pelo hospital.

Os dados coletados de cada paciente internado no período foram: sexo; idade; especialidade médica responsável à internação na UTI; tempo de permanência na unidade; e pontuação diária do NAS, que é realizada pelos enfermeiros do setor, os quais foram treinados para tal fim e executam a mensuração e registro eletrônico do NAS desde 2011.

Ademais, foram extraídas das planilhas da Direção de Enfermagem informações quanto ao quantitativo de trabalhadores por categoria no setor, bem como afastamentos no trabalho. A ausência da medida no NAS, por todo o período de internação do paciente, na planilha de gerenciamento, foi o único critério de exclusão na pesquisa para os pacientes. Já os trabalhadores afastados por qualquer motivo durante o recorte da pesquisa, não foram considerados no quadro de pessoal real (disponível) da unidade.

O NAS é um instrumento de medida da carga de trabalho da enfermagem composto por 32 atividades de avaliação, distribuídas nas seguintes dimensões: atividades básicas, suporte ventilatório, suporte cardiovascular, suporte renal, suporte neurológico, suporte metabólico, e intervenções específicas ${ }^{(12-13)}$. A pontuação máxima do NAS por paciente em 24 horas é de $176,8 \%{ }^{(12-13)}$.

Todas as informações foram armazenadas no software Microsoft Office Excel ${ }^{\circledR}$, versão 2010. Após isso, procedeu-se análise estatística descritiva dos dados,utilizando-se de medidas de proporção, tendência central e dispersão, bem como de equação recomendada para o dimensionamento da equipe de enfermagem em $\mathrm{UTI}^{(14)}$, a saber:

$$
\mathrm{PE}=(\mathrm{E} .(\text { média do NAS } / 100)+15 \%
$$

A notação "PE" significa o quantitativo total de profissionais de enfermagem requeridos (quadro dimensionado) na unidade; "E" o número de equipes disponíveis nos turnos de trabalho, que é multiplicado pela média do NAS da unidade. A média do NAS representou a soma desta pontuação de todos os pacientes em cada um dos 122 dias de análise, dividido por este valor de dias. Esta média foi ainda dividida por 100, pois, segundo a literatura ${ }^{(13)}$, isso deve corresponder a um profissional de enfermagem. Tal resultado foi acrescido de 15\%, representado pelo Índice de Segurança Técnico (IST) mínimo recomendado pela Resolução do COFEN vigente ${ }^{(5)}$ a fim de suprir as ausências previstas e não previstas dos recursos humanos de enfermagem.

A fase de definição qualitativa - proporção de trabalhadores de enfermagem por categoria profissional - do dimensionamento foi obtida também através da Resolução atual (543/2017) do COFEN $^{(5)}$. Para isso, considerou-se a proporção mínima de $52 \%$ de enfermeiros (e os demais, técnicos 
de enfermagem) sobre o total do quadro dimensionado para demanda de pacientes em cuidados intensivos $^{(5)}$, devido à característica do local de pesquisa, ou seja, UTI. Não se considerou a diferença entre cargos de enfermeiros (coordenação versus assistência), sendo estes compilados como uma única categoria profissional, devido à formação pareada.

O estudo cumpriu as exigências éticas do Conselho Nacional de Saúde. Ademais, o projeto de pesquisa que fomentou o estudo foi submetido e aprovado pelo Comitê de Ética em Pesquisa com Seres Humanos da Universidade Estadual do Oeste do Paraná, sob parecer nº 1.450.491.

\section{RESULTADOS}

Em relação à amostra de pacientes investigada, esta foi composta por 128 sujeitos internados na UTI no período de junho a setembro de 2016. Não houve nenhuma perda. A taxa de ocupação na UTI foi de $97,2 \%$. Destarte, a Tabela 1 sumariza os resultados atinentes à caracterização sociodemográfica e clínica dos pacientes.

Tabela 1 - Características sociodemográficas e clínicas dos pacientes (n=128). Cascavel, PR, Brasil, 2016

\begin{tabular}{|c|c|c|}
\hline Variável & Categorias & n (\%) \\
\hline \multirow[t]{2}{*}{ Sexo } & Feminino & $53(41,40)$ \\
\hline & Masculino & $75(58,60)$ \\
\hline \multirow[t]{7}{*}{ Faixa Etária (Anos) } & $15-24$ & $15(11,72)$ \\
\hline & $25-34$ & $18(14,07)$ \\
\hline & $34-44$ & $15(11,72)$ \\
\hline & $45-54$ & $24(18,75)$ \\
\hline & $55-64$ & $27(21,09)$ \\
\hline & $65-74$ & $20(15,62)$ \\
\hline & $75-84$ & $9(7,03)$ \\
\hline \multirow[t]{11}{*}{ Especialidade Médica } & Endocrinologia & $25(19,54)$ \\
\hline & Neurocirurgia & $34(26,57)$ \\
\hline & Cirurgia Geral & $32(25)$ \\
\hline & Cardiologia & $9(7,03)$ \\
\hline & Cirurgia Torácica & $6(4,68)$ \\
\hline & Clínica Médica & $7(5,46)$ \\
\hline & Gastroenterologia & $9(7,03)$ \\
\hline & Cirurgia Plástica & $1(0,78)$ \\
\hline & Bucomaxilo & $1(0,78)$ \\
\hline & Ortopedia e Traumatologia & $3(2,35)$ \\
\hline & Ginecologia e Obstetrícia & $1(0,78)$ \\
\hline \multirow[t]{8}{*}{ Tempo de Internação na UTI (dias) } & $1-4$ & $13(10,16)$ \\
\hline & $5-14$ & $43(33,60)$ \\
\hline & $15-24$ & $27(21,09)$ \\
\hline & $25-34$ & $27(21,09)$ \\
\hline & $35-44$ & $6(4,69)$ \\
\hline & $45-54$ & $5(3,90)$ \\
\hline & $55-64$ & $2(1,57)$ \\
\hline & Mais de 64 dias & $5(3,90)$ \\
\hline
\end{tabular}


A pontuação no NAS dos pacientes de toda a unidade, em cada dia de análise ( $n=122)$, variou de 1258,6 a 2259,0 pontos, obtendo-se a média de 1514,89 pontos ( $D P=500,2)$, que apesar de elevado, acompanhou o tamanho da média e amplitude, ou seja, com dispersão esperada. Com isso, considerando as cinco equipes de trabalho disponíveis na UTI pesquisada e o acréscimo do IST em 15\%, obteve-se o quadro dimensionado total do pessoal de enfermagem em 87 trabalhadores.

No período estudado, a unidade contava com 69 profissionais, sendo destes 11 enfermeiros (um coordenador e 10 enfermeiros assistenciais) e 58 técnicos de enfermagem. Contudo, houve a exclusão de nove técnicos de enfermagem devido a afastamentos e licenças, o que totalizou 60 trabalhadores ativos.

Considerando o ajuste por categoria profissional ao quadro dimensionado do pessoal de enfermagem, conforme Resolução atual do COFEN, a Tabela 2 dispõe a comparação do quadro quantitativo e qualitativo (por categoria profissional) dimensionado de trabalhadores e o real.

Tabela 2 - Comparativo entre os quadros de pessoal de enfermagem dimensionado e real na UTI, por categoria profissional. Cascavel, PR, Brasil, 2016

\begin{tabular}{lccc} 
Quadro de Pessoal & Enfermeiros & Técnicos de Enfermagem & TOTAL \\
\hline Dimensionado & 49 & 38 & 87 \\
\hline Real & 11 & 49 & 60
\end{tabular}

\section{- DISCUSSÃO}

Os dados desta pesquisa apontaram o sexo masculino com predominância de 75 (58,60\%) na internação na UTI. Este achado corrobora com outros estudos que constatam o sexo masculino também predominante, correspondendo a $58 \%$ dos pacientes $(n=104)$ hospitalizados na UTI de um hospital localizado no Rio Grande do Sul ${ }^{(15)}$, e $74 \%$ dos internamentos $(n=50)$ em um centro de tratamento intensivo para queimados ${ }^{(16)}$. Apesar de não ser uma medida considerada no NAS, talvez o sexo da clientela possa refletir subjetivamente na carga de trabalho da equipe de enfermagem, como por exemplo, o peso de pacientes homens comumente superior ao de mulheres, ainda que isso não seja uma regra.

No que se refere à faixa etária, predominaram indivíduos com idade entre 55 a 64 anos, o que também se alia ao observado em outro estudo que apresentou a média de 65,81 anos de idade, no entanto, com predominância do sexo feminino $(71 \%)$ na hospitalização geral ${ }^{(17)}$. Isso reforça que cada serviço, individualmente, incluindo a UTI, deve ter comportamento proativo no conhecimento do perfil de sua clientela, a fim de que as ações de cuidado possam ser planejadas com maior assertividade, incluindo a alocação e a gestão de recursos humanos racionais.

Observa-se que os resultados do presente estudo se assemelham a outras investigações que apresentam a faixa etária de maior predominância entre 50 e 59, seguida de 60 a 69 anos, totalizando $36,1 \%$ dos pacientes assistidos na UTI ${ }^{(18)}$. Por sua vez, na região norte do estado do Paraná, a idade média dos pacientes de cuidados intensivos para pacientes queimados já correspondeu a 41 anos ${ }^{(16)}$.

Os achados quanto à idade dos pacientes, mesmo que não considerados na mensuração da carga de trabalho da enfermagem em si, podem favorecer a alocação estratégica de recursos humanos, uma vez que os pacientes idosos, maior parcela da pesquisa, sabidamente apresentam necessidades de cuidado diferenciadas, ao exemplo de cuidados com a pele, monitoramento da nutrição e hidratação mais intensificados, a fim de prevenir eventos adversos comuns neste setor assistencial (UTI), como as lesões por pressão ${ }^{(19)}$.

Dito isso, alvitra-se que, mesmo que a idade não seja um fator compilado na mensuração da carga de trabalho da enfermagem pelo NAS, ela pode servir de subsídio à gestão de pessoas - fator sobreposto ao dimensionamento de pessoal - como, por exemplo, na capacitação de pessoal em alinhamento ao 
perfil clínico e demográfico da clientela.

A especialidade médica responsável pela internação na UTI mais frequente foi a neurocirurgia, o que pode estar relacionado ao perfil de atendimento da instituição, que é referência em trauma na região. A literatura ${ }^{(20)}$ aponta que doenças do sistema nervoso correspondem a segunda causa de internação em tratamento intensivo, que comumente se desdobram em intervenções cirúrgicas. Neste mesmo estudo, o tempo de internação médio foi de 10 dias, corroborando com os dados desta pesquisa e que podem ter se atrelados à carga de trabalho elevada.

As características clínicas da amostra de pacientes investigada se contrapõem aos achados de uma pesquisa desenvolvida na região nordeste do Brasil, que identificou que a maioria dos internamentos na UTI estava relacionada com doenças cardiorrespiratórias $(46 \%)$ e hepáticas $(17,5 \%)^{(21)}$. Retomando o debate antes expresso, este é um dado que reafirma o perfil da organização hospitalar, ou seja, valendo-se de referência ao trauma em detrimento de enfermidades de manejo clínico, como aquelas vinculadas às especialidades de cardiologia e pneumologia ${ }^{(19)}$, por exemplo, é esperado que a UTI pesquisada vincule-se prioritariamente às internações oriundas de especialidades médicas cirúrgicas, as quais normalmente são responsáveis pelo manejo do paciente politraumatizado, que por condição clínica geral grave, pode demandar aumento de demanda de cuidado pela equipe de enfermagem, traduzindo-se em elevação da carga de trabalho.

No que se refere ao tempo de permanência na UTI, um estudo desenvolvido em Santa Catarina identificou que 53,2\% dos pacientes internados permaneceram por um período de 0 a 3 dias e $29,7 \%$ ficaram de 4 a 15 dias $^{(18)}$. Em uma instituição de ensino, o tempo de permanência na UTI correspondeu em média a 5,46 dias ${ }^{(17)}$. Já no hospital estudado, o período de internação que apresentou maior constância foi de 5 a 14 dias, podendo-se também relacionar ao perfil de saúde da amostra, que, pela gravidade clínica, pode ter apresentado necessidade de maior permanência e, consequentemente, na demanda laboral da enfermagem na unidade.

A UTI em estudo apresentou taxa de ocupação alta (97,2\%). Postula-se que este achado possivelmente se atrela ao fato de que o hospital atende exclusivamente à demanda do SUS, o qual sabidamente sofre com a deficiência de leitos hospitalares, somado a um cenário repleto de adversidades, como a escassez e o sucateamento de recursos das mais diversas ordens, inclusive humanos, como ratifica os dados desta pesquisa. Este é um dado que certamente contribuiu para a elevada carga de trabalho da equipe de enfermagem mensurada pelo NAS diariamente na UTI, que esteve com sua capacidade operacional não raras vezes plenamente ocupada, ou muito próxima a isso.

No que se refere à média de escore do NAS $(1514,89)$ da unidade, pondera-se que a carga de trabalho da equipe de enfermagem foi alta se comparada a estudo desenvolvido em outro hospital universitário do interior do Paraná, cuja média do NAS do setor de tratamento intensivo para adultos apresentada foi de 697,3 pontos $^{(14)}$. Cumpre destacar que a UTI do referido estudo contava apenas com oito leitos de internação, ou seja, próximo à metade da capacidade operacional disponível na unidade investigada. Ainda assim, considerando que o perfil clínico da clientela do estudo citado era semelhante ao desta pesquisa, a carga de trabalho da UTI do hospital situado em Maringá-PR ${ }^{(14)}$, se projetada ao seu dobro, ainda seria inferior à da unidade (de atendimento similar) investigada, situada no mesmo estado e de igual regimento gerencial.

Estudo desenvolvido em unidade de cuidados semi-intensivos para adultos constatou média

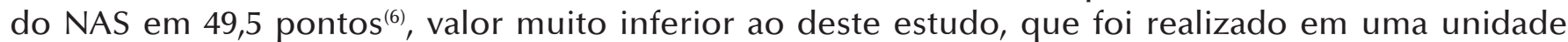
de tratamento intensivo. Isso traz um paralelo de que a clientela internada nesta pesquisa, de fato, apresentava gravidade clínica compatível à internação em UTI, que se desdobrou na alta carga de trabalho da equipe de enfermagem.

Como já explicitado, há constatação de que a influência da carga de trabalho para o desenvolvimento de eventos adversos é real, ao passo que sobrecarga da equipe de enfermagem é um fator de risco ao desenvolvimento de infecções, úlceras por pressão e erros de medicação ${ }^{(9)}$, além de piores resultados entre indicadores relacionados à gestão de recursos humanos, como absenteísmo e rotatividade (turn over) entre os trabalhadores ${ }^{(2)}$.

Ademais, um estudo realizado em 12 cidades europeias mostrou que, para os profissionais de enfermagem, a sobrecarga aliada a jornadas de trabalho longas reflete no desenvolvimento da 
assistência de baixa qualidade, bem como que o desenvolvimento de horas extra em excesso não é uma das soluções mais eficazes para esse problema ${ }^{(22)}$.

A alta carga de trabalho influenciou o quadro dimensionado de pessoal de enfermagem da UTI pesquisada, que apresenta sério déficit (-38) na categoria de enfermeiros. Isso pode ser produto ao fato de que a Resolução vigente do COFEN recomenda que a equipe de enfermagem que presta assistência à pacientes de cuidados intensivos deve ser composta por no mínimo $52 \%$ de enfermeiros ${ }^{(5)}$. Em contraponto, a proporção de enfermeiros no quadro real da UTI era de apenas $18,3 \%$ sobre o total de profissionais.

Aliando a recomendação oficial aos resultados da pesquisa, remete-se à reflexão de que a organização investigada tende a priorizar a contratação de pessoal de nível médio em detrimento àqueles de nível superior, uma vez que a categoria de técnicos de enfermagem obteve um superávit de 11 trabalhadores no quadro dimensionado. Ademais, no quadro real, a proporção dos profissionais de nível médio era de $81,7 \%$, o que remete pleno desacordo à diretriz do COFEN em vigor ${ }^{(5)}$.

O fato antes explícito é comungado por outras pesquisas que realizaram o dimensionamento de enfermagem em $\mathrm{UTI}^{(14,23)}$, ou fora dela, como na sala de emergência de pronto-socorro hospitalar(24). Cumpre refletir sobre a gravidade deste panorama no cenário da enfermagem brasileira, porque sabese que o processo de trabalho do enfermeiro deve permear sinergicamente as ações de gerência para o cuidado direto ${ }^{(3,6)}$, e, quando na deficiência tão expressiva de enfermeiros, estes profissionais talvez possam ter seu trabalho focado em ações burocráticas plenamente desconectadas do planejamento para o melhor cuidado ou até mesmo na prestação direta deste.

A adequação do quadro quantitativo e qualitativo de profissionais, especialmente no contexto dos enfermeiros, pode ser um tabu à ótica puramente organizacional/administrativa, uma vez evidente que este fator incorrerá no dispêndio de recursos financeiros atrelados à folha de pagamento de pessoal com maior renumeração que o de nível técnico. Neste aspecto, um estudo identificou que a adequação de pessoal de enfermagem hospitalar pode representar acréscimo de até $40 \%$ sobre o total pago ao quadro de trabalhadores, independente da categoria ${ }^{(25)}$.

Em contraponto, uma pesquisa recente realizada em hospital universitário de grande porte de Porto Alegre identificou que o incremento de $40 \%$ do número de enfermeiros e $16 \%$ do total de técnicos de enfermagem tem potencial para reduzir sobremaneira os índices de afastamentos por doença, o total do banco de horas excedentes, as horas extras pagas, bem como redução das taxas de lesão por pressão, quedas e infecções relacionadas ao uso de sonda vesical de demora ${ }^{(26)}$.

Considerando o impacto da adequação de capital humano de enfermagem na qualidade e na segurança do paciente, evidencia-se que há necessidade das lideranças do local pesquisado em militar mais ativamente pela contratação de pessoal adicional, em especial enfermeiros. Por se tratar de um hospital público, sabe-se que isso incorre em dificuldades inerentes ao processo de recrutamento e seleção mais moroso, que normalmente se dá via concurso público e/ou testes seletivos com a anuência governamental.

Neste aspecto, espera-se que as entidades de classe da enfermagem possam representar claramente a busca por condições de trabalho melhores no que diz respeito aos recursos humanos, e a atualização da Resolução oficial que rege o dimensionamento de pessoal pode servir como ponte facilitadora a tal empreitada.

\section{- CONCLUSÃO}

Conclui-se que o quadro dimensionado de pessoal de enfermagem na UTI não corresponde ao quadro real apresentado para a categoria de enfermeiros. O déficit de profissionais desta classe foi alto e isso se atrelou ao fato de que a carga de trabalho da unidade foi elevada, que, face à gravidade clínica da clientela e as próprias exigências do COFEN, atribuiu amplo requerimento de cuidados por profissionais com maior nível de capacitação.

Acredita-se que a limitação mais expressiva deste estudo foi considerar apenas o dimensionamento e a carga de trabalho da equipe de enfermagem desvinculados de resultados de aplicabilidade direta 
ao cuidado ou à saúde dos trabalhadores. No entanto, além de possivelmente ser uma das primeiras pesquisas desenvolvidas com a nova Resolução brasileira vigente ao dimensionamento, que é muito recente, o estudo pode contribuir na disseminação do tema à enfermagem nacional e mundial na busca por subsídios de trabalho mais dignos ao cuidado intensivista.

\section{REFERÊNCIAS}

1. Soares MI, Terra FS, Oliveira LS, Resck ZMR, Esteves AMSD, Moura CC. Processo de enfermagem e sua aplicação em unidade de terapia intensiva: revisão integrativa. Rev. enferm. UFPE online. [Internet] 2013;7(n esp) [acessoem 17jan 2017]. Disponível:10.5205/reuol.4134-32743-1-SM-1.0705esp201311.

2. de Magalhães AMM, Dall'Agnol CM, Marck PB. Nursing workload and patient safety - a mixed method study with an ecological restorative approach. Rev. Latino-Am. Enfermagem. [Internet] 2013;21(n. esp) [acesso em 18 jan 2017]. Disponível: http://dx.doi.org/10.1590/S0104-11692013000700019.

3. Vasconcelos RO, Bohrer CD, Rigo DFH, Marques LGS, de Oliveira JLC, Tonini NS, et al. Meios para a gerência de enfermagem utilizados em unidades hospitalares críticas. Enferm. Foco. [Internet] 2016;7(3/4) [acesso em 17 jan 2017].Disponível: http://revista.cofen.gov.br/index.php/enfermagem/article/view/944/354.

4. Chaves LDP, Laus AM, Camelo SH. Ações gerenciais e assistenciais do enfermeiro em unidade de terapia intensiva. Rev. Eletr. Enferm.[Internet] 2012;14(3) [acesso em 17 jan 2017]. Disponível: http://dx.doi.org/10.5216/ ree.v14i3.15724.

5. Conselho Federal de Enfermagem. Resolução n.543/2017. Atualiza e estabelece parâmetros para o Dimensionamento do Quadro de Profissionais de Enfermagem nos serviços/locais em que são realizadas atividades de enfermagem. [Internet] Brasília: COFEN; 2017 [acesso em 24 mai 2017]. Disponível: http://www. cofen.gov.br/resolucao-cofen-5432017_51440.html.

6. Trettene AS, Luiz AG, Razera APR, Maximiano TO, Cintra FMRN, Monteiro LM. Carga de trabalho de enfermagem em Unidade de Terapia Semi-intensiva especializada: critérios para dimensionamento de pessoal. Rev.esc. enferm. USP. [Internet] 2015;49(6)[acesso em 17 jan 2017]. Disponível: http://dx.doi.org/10.1590/S0080623420150000600012.

7. Ferreira PC, Machado RC, Vitor AF, Lira ALBC, Martins QCS. Nursing measure in Intensive Care Unit: evidence about the Nursing Activities Score. Rev. Rene. [Internet] 2014;15(5) [acesso em 03 mai 2017]. Disponível: http:// www.revistarene.ufc.br/revista/index.php/revista/article/view/1685/pdf_1.

8. Novaretti MCZ, Santos EV, Quitério LM, Daud-Gallottil RM. Sobrecarga de trabalho da enfermagem e incidentes e eventos adversos em pacientes internados em UTI. Rev.bras. enferm. [Internet] 2014;67(5) [acesso em 17 jan 2017].Disponível: http://dx.doi.org/10.1590/0034-7167.2014670504.

9. de Oliveira AC, Garcia PC, Nogueira LS. Nursing workload and occurrence of adverse events in intensive care: a systematic review. Rev.esc.enferm. USP. [Internet] 2016;50(4)[acesso em 17 jan 2017]. Disponível: http://dx.doi. org/10.1590/S0080-623420160000500020.

10.Neuraz A, Guérin C, Payet C, Polazzi S, Aubrun F, Dailler F,et al.Patient mortality is associated with staff resources and workload in the ICU: a multicenter observational study.CritCareMed. [Internet]2015;43(8) [acessoem 10 jan 2017]. Disponível: http://dx.doi.org/10.1097/CCM.0000000000001015.

11. Perão OF, BubMBC, Rodríguez AH, Zandonadi GC. Gravidade de pacientes e carga de trabalho de enfermagem em unidade de terapia intensiva. CogitareEnferm. [Internet] 2014;19(2) [acesso em 10 jan 2017].Disponível:http:// dx.doi.org/10.5380/ce.v19i2.33750.

12. Queijo AF, Padilha KG. NursingActivities Score (NAS): adaptação transcultural e validação para a língua portuguesa. Rev. esc. enferm. USP. [Internet] 2009;43(n. esp) [acesso em 10 jan 2017]. Disponível: http://dx.doi. org/10.1590/S0080-62342009000500004.

13. Miranda DR, Nap R, Rijk A, Schaufeli W, lapichino G, TISS Working Group. Therapeutic Intervention Scoring System. NursingActivities Score (NAS). CritCare Med. [Internet] 2003;31(2)[acesso em 17 jan 2017]. Disponível: http://dx.doi.org/10.1097/01.CCM.0000045567.78801.CC. 
14. Inoue KC, Matsuda LM. Dimensionamento de pessoal de enfermagem em Unidade de Terapia Intensiva para adultos. Acta paul. enferm. [Internet] 2010;23(3)[acesso em 10 jan 2017]. Disponível: http://dx.doi.org/10.1590/ S0103-21002010000300011.

15. Favarin SS, Camponogara S. Perfil dos pacientes internados na unidade de terapia intensiva adulto de um hospital universitário. Rev enferm. UFSM.[Internet] 2012;2(2) [acesso em 10 jan 2017]. Disponível: http://dx.doi. org/10.5902/217976925178.

16. Camuci MB, Martins JT, Cardeli AAM, Robazzi MLCC.NursingActivities Score: carga de trabalho de enfermagem em Unidade de Terapia Intensiva de queimados. Rev. Latino-Am. Enferm. [Internet] 2014;22(2) [acesso em10 jan 2017]. Disponível: http://dx.doi.org/10.1590/0104-1169.3193.2419.

17. Bezerra GKA. Unidade de Terapia Intensiva - Perfil das Admissões: Hospital Regional de Guarabira, Paraíba, Brasil. R bras ci Saúde. [Internet] 2012;16(4) [acesso em 28 dez 2016]. Disponível: http://www.ies.ufpb.br/ojs/ index.php/rbcs/article/viewFile/11900/9116.

18. Rodriguez AH, Bub MBC, Perão OF, Zandonadi G, Rodriguez MJH. Epidemiological characteristics and causes of deaths in hospitalized patients under intensive care.Rev. bras. enferm. [Internet] 2016;69(2) [acesso em 23 dez 2016]. Disponível: http://dx.doi.org/10.1590/0034-7167.2016690204i.

19. Fernandes LM, da Silva L, de Oliveira JLC, de Souza VS, Nicola AL. Associação entre predição para lesão por pressão e marcadores bioquímicos.Rev. Rene. [Internet] 2016;17(4) [acesso em 18 jan 2017]. Disponível: http:// www.periodicos.ufc.br/index.php/rene/article/view/4939/3640.

20. Melo ACL, Menegueti MG, Laus AM. Perfil de pacientes de terapia intensiva: subsídios para a equipe de enfermagem. Rev. enferm. UFPE online. [Internet] 2014;8(9) [acesso em 15 dez 2016]. Disponível: http://www. revista.ufpe.br/revistaenfermagem/index.php/revista/article/viewFile/4912/pdf_6129.

21. da Silva LS, Mont'alverne DGB, de Medeiros AIC, da Silva AGCB, Carvalho EM. Características dos pacientes sob assistência fisioterapêutica na UTI de um hospital universitário: estudo epidemiológico transversal. Rev Fisioter S Fun. Fortaleza. [Internet] 2015;5(1) [acesso em 23 dez 2016]. Disponível: http://www.fisioterapiaesaudefuncional. ufc.br/index.php/fisioterapia/article/view/627/pdf.

22. Griffiths P, Dall'ora C, Simon M, Ball J, Linqvist R, Rafferty A, et al. Nurses' shift lengthandovertimeworking in 12 European countries: theAssociationwithperceivedqualityofcareandpatientsafety. MedCare. [Internet] 2014;52(11) [acesso em 21 dez 2016]. Disponível:http://dx.doi.org/10.1097/MLR.0000000000000233.

23. Pias C, Mascolo NP, da Silva ERR, Linch GFC, de Souza EM. Complexidade da assistência em unidade de terapia intensiva: subsídios para dimensionamento de pessoal de enfermagem.CogitareEnferm. [Internet] 2015;20(3) [acesso em 28 dez 2016]. Disponível: http://dx.doi.org/10.5380/ce.v20i3.41083.

24. da Paixão TCR, Campanharo CRV, Lopes MCBT, Okuno MFP, Batista REA. Nursing staff sizing in the emergency room of a university hospital. Rev. esc. enferm. USP. [Internet] 2015;49(3).[acesso em 18 jan 2017]. Disponível: http://dx.doi.org/10.1590/S0080-623420150000300017.

25. de Araújo TR, Menegueti MG, Auxiliadora-Martins M, Castilho V, Chaves LDP, Laus AM. Impacto financeiro do quadro de profissionais de enfermagem requerido em Unidade de Terapia Intensiva. Rev. Latino-Am. Enfermagem. [Internet] 2016;24(n. esp) [acesso em 18 jan 2017].Disponível:http://dx.doi.org/10.1590/1518-8345.1274.2818.

26. de Quadros DV, de Magalhães AMM, Mantovani VM, da Rosa DS, Echer IC. Analysis of managerial and healthcare indicators after nursing personnel upsizing.Rev.bras. enferm. [Internet] 2016;69(4)[acesso em 18 jan 2017]. Disponível: http://dx.doi.org/10.1590/0034-7167.2016690410i. 